\title{
ASTHENO-KÄHLER AND BALANCED STRUCTURES ON FIBRATIONS
}

\author{
ANNA FINO, GUEO GRANTCHAROV AND LUIGI VEZZONI
}

\begin{abstract}
We study the existence of three classes of Hermitian metrics on certain types of compact complex manifolds. More precisely, we consider balanced, SKT and astheno-Kähler metrics. We prove that the twistor spaces of compact hyperkähler and negative quaternionic-Kähler manifolds do not admit astheno-Kähler metrics. Then we provide a construction of astheno-Kähler structures on torus bundles over Kähler manifolds leading to new examples. In particular, we find examples of compact complex non-Kähler manifolds which admit a balanced and an astheno-Kähler metrics, thus answering to a question in [52 (see also 24]). One of these examples is simply connected. We also show that the Lie groups $S U(3)$ and $G_{2}$ admit SKT and astheno-Kähler metrics, which are different. Furthermore, we investigate the existence of balanced metrics on compact complex homogeneous spaces with an invariant volume form, showing in particular that if a compact complex homogeneous space $M$ with invariant volume admits a balanced metric, then its first Chern class $c_{1}(M)$ does not vanish. Finally we characterize Wang C-spaces admitting SKT metrics.
\end{abstract}

\section{INTRODUCTION}

After it became clear that certain complex manifolds do not admit Kähler metrics, the question of finding appropriate generalizations naturally arose. Although a universal type of Hermitian non-Kähler metrics has not been found yet, several classes, related to different geometric or physics applications, have been introduced and studied. The present paper focuses on the existence and interplay between three such classes: astheno-Kähler, SKT and balanced metrics on particular examples of compact complex non-Kähler manifolds.

A Hermitian metric $g$ on a complex manifold $(M, I)$ is called astheno-Kähler if its fundamental form $F(\cdot, \cdot):=g(I \cdot, \cdot)$ satisfies

$$
d d^{c} F^{n-2}=0,
$$

Date: February 8, 2018.

2000 Mathematics Subject Classification. Primary 53C15 ; Secondary 53C55, 53C30.

Key words and phrases. astheno-Kähler, balanced, complex homogeneous space.

The work of the first and third authors was supported by the project FIRB "Geometria differenziale e teoria geometrica delle funzioni" and by G.N.S.A.G.A. of I.N.d.A.M. The work of the second author is supported by the Simons Foundation grant \#246184. 
where $n$ is the complex dimension of $M, d^{c}=I^{-1} d I$ and $I$ is naturally extended on differential forms. Such metrics were used by Jost and Yau in [40] to establish existence of Hermitian harmonic maps which led to some information about the fundamental group of the targets. As application, Carlson and Toledo made use of astheno-Kähler metrics to get restrictions on the fundamental group of complex surfaces of class VII [16]. Later harmonic maps from more general Hermitian manifolds have been studied in [44]. In [43] Li, Yau, and Zheng conjectured that compact non-Kähler Hermitianflat manifolds or similarity Hopf manifolds of complex dimension $\geq 3$ do not admit any astheno-Kähler metric. Moreover, Tosatti and Weinkove proved in [56] CalabiYau theorems for Gauduchon and strongly Gauduchon metrics on the class of compact astheno-Kähler manifolds. Until recently astheno-Kähler metrics were not receiving a lot of attention due to the lack of examples. Indeed, there are not many examples of astheno-Kähler manifolds, some of them are given by Calabi-Eckmann manifolds [45] and by nilmanifolds [28]. In the present paper we provide a construction which leads to many new ones.

We further recall that a Hermitian metric $g$ on a complex manifold $(M, I)$ is called strong Kähler with torsion (SKT) or pluriclosed, if its fundamental form $F$ satisfies $d d^{c} F=0$, while it is called balanced if $F$ is co-closed. Moreover, $g$ is called standard (or Gauduchon) if $d d^{c} F^{n-1}=0$. For $n=3$ the notions of astheno-Kähler and SKT metric coincide, while in higher dimensions they lie in different classes. The study of SKT metrics was initiated by Bismut in [10] and then it was pursued in many papers (see e.g. [33, 50, 26, 17] and the references therein). For a complex surface the notions of SKT metric and standard metric coincide and in view of [34] every compact complex surface has an SKT metric. In higher dimensions things are different and there are known examples of complex manifolds not admitting SKT metrics. For instance, Verbitsky showed in [58] that the twistor space $M$ of a compact, antiselfdual Riemannian manifold admits an SKT metric if and only it is Kähler (hence if and only if it is isomorphic to $\mathbb{C P}^{3}$ or to a flag space). This result is obtained by using rational connectedness of twistor spaces, proved by Campana in [15].

Examples of compact SKT manifolds are provided by principal torus bundles over compact Kähler manifolds [36], by nilmanifolds [26, 57, 23], and other examples can be constructed by using twist construction [51] or blow-ups [27.

Balanced geometry is probably the most studied, partly due to its relation with string theory and Strominger's system. The terminology was introduced by Michelsohn in [47, where balanced metrics were first studied in depth. In particular, Michelsohn showed an obstruction to the existence of balanced metrics by using currents. From Michelsohn's obstruction it follows that Calabi-Eckmann manifolds have no balanced metrics. Balanced metrics are stable under modifications, but not under deformations. Basic examples of balanced manifolds are given by twistor spaces. First it was known that twistor spaces of selfdual 4-manifolds are balanced [47] and later 
the same was proven for twistor spaces of a hyperkähler (see [41]) and quaternionicKähler manifolds ([48, 4]) and, most recently, by the twistor spaces of a compact hypercomplex manifold [55]. Taubes in [54] used the twistor space examples to show that every finitely generated group is a fundamental group of a balanced manifold.

It is known that a Hermitian metric on a compact manifold is simultaneously balanced and SKT if and only if it is Kähler (see e.g. [5]). Furthermore, it was proved that many classes of examples of balanced manifolds do not admit any SKT metric [19, 20, 29, 31, 58, 30].

This led Fino and Vezzoni to ask in [29] whether there are compact complex manifolds admitting both SKT and balanced metrics but which admit no Kähler structure. In the same spirit, one can wonder if a compact complex non-Kähler manifold could admit both an astheno-Kähler and a balanced metric. This second problem arises from [52, where it is proved that the Calabi-Yau type equation introduced in [32] is solvable on astheno-Kähler manifolds admitting balanced metrics. There was an opinion, formulated as part of a folklore conjecture in [24], that as in the SKT case, a balanced compact complex manifold can not admit an astheno-Kähler, unless it is Kähler. Note that, as in the SKT case, a metric on a compact manifold cannot be both balanced and astheno-Kähler simultaneously, unless Kähler.

In the present paper (Section 4), using the general construction of Section 3, we construct an example of an 8-dimensional non-Kähler principal torus bundle over a torus admitting both an astheno-Kähler metric and a balanced metric. It is also a 2-step nilmanifold with holomorphically trivial canonical bundle. Examples of nilmanifolds in every dimension $2 n \geq 8$ have been constructed independently by Latorre and Ugarte [42. In Section 5 we also find a simply connected compact example of dimension 22 with non-vanishing first Chern class. It is the complex homogeneous space $S U(5) / T^{2}$ with a complex structure studied by H. C. Wang [59].

In Section 2 we show that twistor spaces over compact hyperkähler manifolds and twistor spaces over compact quaternion-Kähler manifolds cannot admit asthenoKähler metrics. We prove this result by providing a new obstruction, which generalizes the one in [40], to the existence of astheno-Kähler metrics by using currents in the spirit of [47]. Furthermore, in Section 3 we provide a construction of astheno-Kähler metrics on some principal torus bundles over Kähler manifolds generalizing the result of Matsuo for Calabi-Eckmann manifolds [45]. The construction could be used to find many new examples. Apart from Section 4, we use it to find astheno-Kähler metrics on the Lie groups $S U(3)$ and $G_{2}$. The result gives examples of compact complex non-Kähler manifolds admitting both an astheno-Kähler and an SKT metric. In the last part of the paper we investigate the existence of balanced metrics on compact complex manifolds with an invariant volume form. Compact complex homogeneous spaces with invariant volumes have been classified in [37], showing that every compact complex homogeneous space with an invariant volume form is a principal homogeneous complex torus bundle over the product of a projective rational homogeneous 
space and a complex parallelizable manifold. We obtain a characterisation of the balanced condition in terms of the characteristic classes of the associated torus fibration and of the Kähler cone of the projective rational homogeneous space. As a consequence we show that if a compact complex homogeneous space $M$ with invariant volume admits a balanced metric, then its first Chern class is non-vanishing.

Finally, in the last section we study the existence of SKT metrics on Wang Cspaces, i.e. on compact complex non-Kähler manifolds with finite fundamental group, admitting a transitive action by a compact Lie group of biholomorphisms. We show that every Wang C-space admitting an SKT metric can be covered with a product of a compact Lie group and a generalized flag manifold. In particular our example $S U(5) / T^{2}$ cannot admit SKT metrics.

\section{NON-EXISTENCE OF ASTHENO-KÄHLER METRICS ON TWISTOR SPACES}

In this section we show that twistor spaces of hyperkähler and quaternionic-Kähler manifolds do not admit any astheno-Kähler metric.

A hyperkähler manifold is a Riemannian manifold $(M, g)$ with holonomy contained in $S p(n)$. The hyperkähler condition can be characterized by the existence of three complex structures $I, J, K$ each one inducing a Kähler structure with $g$ and satisfying the quaternionic relation $I J=-J I=K$. As a consequence, for any $p=(a, b, c) \in$ $S^{2}, a^{2}+b^{2}+c^{2}=1$, the endomorphism $I_{p}=a I+b J+c K$ is an integrable almost complex structure. The twistor space $T w(M)$ of $M$ is then defined as the space $T w(M)=M \times S^{2}$ endowed with the tautological complex structure $\left.\mathcal{I}\right|_{(x, p)}=\left.I_{p}\right|_{T_{x} M} \times$ $I_{S^{2}}$. Note that there are two natural projections $\pi_{1}: T w(M) \rightarrow M$ and $\pi_{2}: T w(M) \rightarrow$ $S^{2}$ where the second one is holomorphic, but the first one is not.

A Riemannian manifold $(M, g)$ is called quaternionic-Kähler if its holonomy is in $S p(n) S p(1)$. In analogy to the hyperkähler case, the quaternionic-Kähler condition can be characterized by the existence of a parallel sub-bundle $D$ of $\operatorname{End}(T M)$ locally spanned by a triple of almost complex structures $I, J, K$, each one compatible with $g$ and satisfying the quaternionic relations $I J=-J I=K$. Although the definition of quaternionic-Kähler manifold is similar to the one of hyperkähler manifold, the geometric properties of these two kind of manifolds are rather different. One common feature, however is the existence of a twistor space. The twistor space $Q(M)$ of a quaternionic-Kähler $(M, g)$ is defined as the $S^{2}$-bundle over $M$ with fiber the 2-sphere in $D$ naturally identified with the set of almost complex structures $S=\{a I+b J+$ $\left.c K \mid a^{2}+b^{2}+c^{2}=1\right\}$. Although $I, J, K$ are locally defined, $S$ doesn't depend on their choice. The twistor space $Q(M)$ has a tautological almost complex structure $\mathcal{I}$, similar to the one defined in the hyperkähler case. It uses a splitting $T Q(M)=H \oplus T_{S^{2}}$, where $H$ is a horizontal subspace, defined via the Levi-Civita connection of $g$ which defines one on $D$. It is known that $\mathcal{I}$ is integrable and the metric $g_{Q}=\left.\pi^{*} g\right|_{H} \oplus t g_{S^{2}}$ is Hermitian for every positive $t$. Hyperkähler manifolds are Ricci-flat, while the quaternionic-Kähler ones are Einstein. In particular quaternionic-Kähler metrics have 
constant scalar curvature and are called of positive or negative type depending on their sign. For a positive quaternionic-Kähler manifold $M$, the metric $g_{Q}$ and the structure $\mathcal{I}$ define a Kähler structure for an appropriate choice of $t$. In other words, if $W$ is the fundamental form of $g$ and $\mathcal{I}, d W=0$ for some $t$. In [21] one finds a computation of $d d^{c} W$, from this computation it follows that $d d^{c} W$ is weakly positive if the scalar curvature of the base is negative.

In order to show that twistor spaces do not admit astheno-Kähler metrics, we prove that they are obstructed. In [40] Jost and Yau show that on a compact complex manifold admitting an astheno-Kähler metric every holomorphic 1-form is closed, giving an obstruction to the existence of astheno-Kähler metrics. We generalize the obstruction of Jost and Yau in the spirit of [39].

Here we recall that a $(p, p)$-current on a complex manifold $(M, I)$ is an element of the Frechet space dual to the space of $(n-p, n-p)$ complex forms $\Lambda^{n-p, n-p}(M)$. In the compact case, the space of $(p, p)$-currents can be identified with the space of $(p, p)$-forms with distribution coefficients and the duality is given by integration. So for any $(p, p)$-current $T$ and a form $\alpha$ of type $(n-p, n-p)$ we have

$$
\langle T, \alpha\rangle=\int_{M} T \wedge \alpha
$$

The operators $d$ and $d^{c}$ can be extended to $(p, p)$-currents by using the duality induced by the integration, i.e., $d T$ and $d^{c} T$ are respectively defined via the relations

$$
\langle d T, \beta\rangle=-\int_{M} T \wedge d \beta, \quad\left\langle d^{c} T, \beta\right\rangle=-\int_{M} T \wedge d^{c} \beta .
$$

A $(p, p)$-current $T$ is called weakly positive if

$$
i^{n-p} \int_{M} T \wedge \alpha_{1} \wedge \overline{\alpha_{1}} \wedge \ldots \alpha_{n-p} \wedge \bar{\alpha}_{n-p} \geq 0
$$

for every (1,0)-forms $\alpha_{1}, \ldots \alpha_{n-p}$ with inequality being strict for at least one choice of $\alpha_{i}$ 's. The current $T$ is called positive if the inequality is strict for every non-zero $\alpha_{1} \wedge \overline{\alpha_{1}} \wedge \ldots \alpha_{n-p} \wedge \bar{\alpha}_{n-p}$. From [6, Theorem 2.4] (with an obvious change) we have the following:

Theorem 2.1. A compact complex manifold admits a positive $d d^{c}$-closed $(p, p)$-form if and only if it does not admit a $d d^{c}$-exact weakly positive and non-zero $(n-p, n-p)$ current.

Since $F^{n-2}$ is positive, we have the following useful corollary, to which we provide an independent proof.

Corollary 2.1. If a compact complex manifold $M$ admits a weakly positive and $d d^{c}$ exact and non-vanishing $(2,2)$-current, then it does not admit an astheno-Kähler metric. 
Proof. Suppose $d d^{c} T$ is weakly positive, where we consider $T$ as a form with distribution coefficients. Then for an astheno-Kähler metric with fundamental form $F$ we have by integration by parts

$$
0<\int_{M} d d^{c} T \wedge F^{n-2}=\int_{M} T \wedge d d^{c}\left(F^{n-2}\right)=0,
$$

which gives a contradiction.

Note that for a holomorphic 1-form $\alpha$, the form $i \partial \alpha \wedge \overline{\partial \alpha}=d d^{c}(\alpha \wedge \bar{\alpha})$ is weakly positive, which leads to the obstruction of Jost and Yau. Furthermore, we remark that the statement of Corollary 2.1 cannot be reversed, since in general not every positive $(n-2, n-2)$-form arises as $(n-2)$-power of a positive $(1,1)$-form.

Then we can prove the non-existence of an astheno-Kähler metric on twistor spaces.

Proposition 2.1. The twistor space Tw(M) of a compact hyperkähler manifold does not admit any astheno-Kähler metric, compatible with the tautological complex structure.

Similarly, the twistor space $Q(M)$ of a compact quaternionic-Kähler manifold of negative scalar curvature does not admit an astheno-Kähler metric, compatible with the tautological complex structure.

Proof. Let $(M, I, J, K, g)$ be a compact hyperkähler manifold and let $T w(M)=$ $M \times S^{2}$ be its twistor space. Then $G=g_{M}+g_{S^{2}}$ gives a Hermitian metric on $T w(M)$, compatible with the tautological complex structure. We denote by $W$ the fundamental form of $G$. In view of [41], given $(p, r) \in T w(M)$ one has

$$
d d^{c} W_{(p, r)}=F_{p} \wedge \omega_{r}
$$

where $F_{p}(X, Y)=g\left(I_{p} X, Y\right)$ for tangent vectors $X, Y \in T_{p} M$, and $\omega$ is the FubiniStudy form on $S^{2} \equiv \mathbb{C P}^{1}$. Therefore $d d^{c} W$ is a weakly-positive non-vanishing $(2,2)$ current and Corollary 2.1 implies that $T w(M)$ has not astheno-Kähler metrics.

About the quaternion-Kähler case, using the splitting $T Q(M)=H \oplus T S^{2}$ one can define the 1-parameter family of $\mathcal{I}$-compatible Hermitian metrics $g_{Q}=\left.\pi^{*} g\right|_{H} \oplus t g_{S^{2}}$, where $t$ is a positive parameter.

Let us denote by $W_{Q}$ the fundamental of form of $g_{Q}$. Then in view of [21, Theorem $5.4]$ if the scalar curvature of $g$ is negative, then $d d^{c} W_{Q}$ is weakly positive and, consequently, the Corollary 2.1 can be applied also in this case and the claim follows.

\section{AsthenO-KÄHLER METRICS ON TORUS BUNDLES}

In [45] Matsuo showed the existence of astheno-Kähler metrics on Calabi-Eckmann manifolds. Since Calabi-Eckmann manifolds are principal $T^{2}$-bundles over $\mathbb{C P}^{n} \times \mathbb{C P}^{m}$, it is quite natural to extend the Matsuo's result to principal torus fibrations over compact Kähler manifolds. 
Proposition 3.1. Let $\pi: P \rightarrow M$ be an $n$-dimensional principal torus bundle over a Kähler manifold $(M, J, F)$ equipped with 2 connections 1 -forms $\theta_{1}, \theta_{2}$ whose curvatures $\omega_{1}, \omega_{2}$ are of type $(1,1)$ and are pull-backs from forms $\alpha_{1}$ and $\alpha_{2}$ on $M$. Let $I$ be the complex structure on $P$ defined as the pull-back of $J$ to the horizontal subspaces and as $I\left(\theta_{1}\right)=\theta_{2}$ along vertical directions. Let $\Omega=\pi^{*}(F)+\theta_{1} \wedge \theta_{2}$; then

$$
d d^{c} \Omega^{k}=k\left(\omega_{1}^{2}+\omega_{2}^{2}\right) \wedge\left(\pi^{*}\left(F^{k-1}\right)\right), \quad 1 \leq k \leq n-2 .
$$

In particular if $\left(\alpha_{1}^{2}+\alpha_{2}^{2}\right) \wedge F^{n-3}=0$, then $\Omega$ is astheno-Kähler and if a torus bundle with 2-dimensional fiber over a Kähler base admits an SKT metric, then it is asthenoKähler.

Proof. Since $d \theta_{i}=\omega_{i}$ and $d F=0$, we have

$$
d \Omega=\pi^{*}(d F)+\omega_{1} \wedge \theta_{2}-\theta_{1} \wedge \omega_{2}=\omega_{1} \wedge \theta_{2}-\theta_{1} \wedge \omega_{2},
$$

which implies

$$
d^{c} \Omega=\omega_{1} \wedge \theta_{1}+\theta_{2} \wedge \omega_{2} .
$$

Therefore

$$
d d^{c} \Omega=\omega_{1}^{2}+\omega_{2}^{2}
$$

and

$$
d \Omega \wedge d^{c} \Omega=-\left(\omega_{1}^{2}+\omega_{2}^{2}\right) \wedge \theta_{1} \wedge \theta_{2} .
$$

This leads to the following simplified expression for $d d^{c} \Omega^{n-2}$

$$
\begin{aligned}
d d^{c} \Omega^{k} & =k d\left(d^{c} \Omega \wedge \Omega^{k-1}\right) \\
& =k\left(d d^{c} \Omega \wedge \Omega+(k-1) d \Omega \wedge d^{c} \Omega\right) \wedge \Omega^{k-2} \\
& =k\left(\omega_{1}^{2}+\omega_{2}^{2}\right) \wedge\left(\pi^{*}(F)+\theta_{1} \wedge \theta_{2}-(k-1) \theta_{1} \wedge \theta_{2}\right) \wedge \Omega^{k-2} \\
& =k\left(\omega_{1}^{2}+\omega_{2}^{2}\right) \wedge\left(\pi^{*}(F)-(k-2) \theta_{1} \wedge \theta_{2}\right) \wedge \Omega^{k-2} .
\end{aligned}
$$

Moreover we have

$$
\begin{aligned}
\Omega^{k-2}=\left(\pi^{*}(F)+\theta_{1} \wedge \theta_{2}\right)^{k-2} & =\pi^{*}\left(F^{k-2}\right)+(k-2) \theta_{1} \wedge \theta_{2} \wedge \pi^{*}\left(F^{k-3}\right) \\
& =\left(\left(\pi^{*}(F)+(k-2) \theta_{1} \wedge \theta_{2}\right)\right) \wedge \pi^{*}\left(F^{k-3}\right),
\end{aligned}
$$

so after substitution we get

$$
d d^{c} \Omega^{k}=k\left(\omega_{1}^{2}+\omega_{2}^{2}\right) \wedge\left(\pi^{*}\left(F^{k-1}\right)\right),
$$

as required.

Remark 3.1. The situation is similar to the ones in the examples of nilmanifolds obtained in [28]. Proposition 3.1 includes the case of Calabi-Eckmannn manifolds studied in [45]. In the Calabi-Eckmann case we have $\omega_{1}=\Phi_{1}+a \Phi_{2}$ and $\omega_{2}=b \Phi_{2}$, where $\Phi_{1}$ and $\Phi_{2}$ are the Fubini-Study forms on the factors. Note that the metric in [45] is not SKT. In fact to construct examples of astheno-Kähler structures we only need to find closed $(1,1)$-forms $\omega_{1}$ and $\omega_{2}$ such that $\omega_{1}^{2} \wedge F^{n-2}$ and $\omega_{2}^{2} \wedge F^{n-2}$ are constant multiples of the volume form with different signs. This is achieved by taking appropriate linear combinations of $\theta_{1}$ and $\theta_{2}$. 


\section{Interplay BetweEn SPECial Classes of HeRmitian METRICS}

A problem in complex non-Kähler geometry is to establish if the existence of two Hermitian metrics belonging two different classes on a compact complex manifold impose some restrictions.

In 29] Fino and Vezzoni conjectured that the existence of a balanced and an SKT metric on a compact complex manifold $(M, I)$, forces $M$ to be Kähler. Analogue problems make sense by replacing the SKT and the balanced assumption with other different classes of Hermitian metrics.

In this section we provide examples of compact manifolds admitting both SKT and astheno-Kähler metrics and an example having both balanced and astheno-Kähler metrics.

Basic examples of SKT manifolds are given by the compact Lie groups endowed with the Killing (biinvariant) metric and any of the compatible Samelson's complex structures [49]. Samelson's construction depends on a choice of the maximal torus and such complex structures are compatible if they are compatible with the metric restricted to this torus. Let $G$ be a simple compact Lie group and $T^{n}$ be one of its maximal toral subgroups, so that $F l=G / T^{n}$ is a flag manifold. Assume that $n$ is even. Then the projection $\pi: G \rightarrow F l$ (called Tits fibration) is as above and is holomorphic for the Samelson's complex structures. However the metric induced on $F l$ from the Killing metric on $G$ is not Kähler. The Kähler ones arise from forms on adjoint orbits (see [9]). We use the relation between the two metrics in the following proposition.

Proposition 4.1. A compact semisimple Lie group of even dimension $2 n>6$ and of rank two endowed with its Samelson's complex structure admits an astheno-Kähler non-SKT metric while its canonical SKT metric is not astheno-Kähler. In particular, the Lie groups $S U(3)$ and $G_{2}$ admit astheno-Kähler metrics.

Proof. Let $G$ be a compact Lie group of real dimension $2 n$ and let $T^{2}$ be the maximal torus of $G$.

Let $\pi: G \rightarrow G / T^{2}=F l$ be the holomorphic projection onto the corresponding flag manifold as mentioned above. Let $\alpha_{1}$ and $\alpha_{2}$ be invariant representatives of the characteristic classes of $\pi$ and $g_{1}$ be the bi-invariant metric on $G$. We suppose that the metric $g_{1}$ is compatible with the complex structure on $G$ so that if $\Omega_{1}$ is the fundamental form, it is well-known that $d d^{c} \Omega_{1}=0$. Moreover $g_{1}$ induces a naturallyreductive metric on $F l$ with form $F_{1}$ and such that $\Omega_{1}=F_{1}+\theta_{1} \wedge \theta_{2}$, where $\theta_{1}$ and $\theta_{2}$ are orthonormal invariant 1 -forms on $G$ with respect to $g_{1}$ and $I\left(\theta_{1}\right)=\theta_{2}$. Then $d \theta_{i}=\omega_{i}$ for $i=1,2$, where $\omega_{i}$ are independent linear combinations of $\alpha_{i}$. We can always achieve this, since there are invariant 1 -forms $\bar{\theta}_{i}$ with $d \bar{\theta}_{i}=\alpha_{i}$. This is an example of the torus bundle construction from the previous section and we have

$$
0=d d^{c} \Omega_{1}=\pi^{*}\left(d d^{c} F_{1}\right)+\omega_{1}^{2}+\omega_{2}^{2}
$$


i.e.,

$$
\omega_{1}^{2}+\omega_{2}^{2}=-d d^{c}\left(\pi^{*}\left(F_{1}\right)\right) .
$$

Now we consider the Kähler-Einstein metric $g_{2}$ on $F l$ and denote by $F_{2}$ its fundamental form. Let $\Omega_{2}$ be the Hermitian form on $G$ defined as

$$
\Omega_{2}=\pi^{*}\left(F_{2}\right)+\theta_{1} \wedge \theta_{2}
$$

We next show that $d d^{c} \Omega_{2}^{n-2}=0$. By equation (??), $\Omega_{2}^{n-2}$ is $d d^{c}$-closed if and only if

$$
\left(\omega_{1}^{2}+\omega_{2}^{2}\right) \wedge F_{2}^{n-3}=0 \text {. }
$$

But (1) implies

$$
\left(\omega_{1}^{2}+\omega_{2}^{2}\right) \wedge F_{2}^{n-3}=-d d^{c} F_{1} \wedge F_{2}^{n-3}=-d\left(d^{c} F_{1} \wedge F_{2}^{n-3}\right) .
$$

Hence $\left(\omega_{1}^{2}+\omega_{2}^{2}\right) \wedge F_{2}^{n-3}$ is exact on $F l$. It is also invariant, so it is constant multiple of the volume form. Since its integral is zero it vanishes which implies that $\Omega_{2}^{n-2}$ is $d d^{c}$-closed.

Since $S U(3)$ and $G_{2}$ are even-dimensional compact (semi)simple Lie groups of rank two, the proposition is proved.

Note that the SKT form $\Omega_{1}$ is not astheno-Kähler and $\Omega_{2}$ is not SKT. Metrics which are both SKT and astheno-Kähler are constructed in [28].

Example 4.1. Next we provide an example of a compact 8-dimensional complex manifold admitting a balanced and an astheno-Kähler metric. The example is the total space of a principal $T^{2}$-bundle over a 6-dimensional torus. Let $\pi: M \rightarrow T^{6}$ be the principal $T^{2}$ bundle over $T^{6}$ with characteristic classes

$$
a_{1}=d z_{1} \wedge d \bar{z}_{1}+d z_{2} \wedge d \bar{z}_{2}-2 d z_{3} \wedge d \bar{z}_{3}, \quad a_{2}=d z_{2} \wedge d \bar{z}_{2}-d z_{3} \wedge d \bar{z}_{3},
$$

where $\left(z_{1}, z_{2}, z_{3}\right)$ are complex coordinates on $T^{6}$. Consider on $T^{6}$ the standard complex structure and let

$$
F_{1}=d z_{1} \wedge d \bar{z}_{1}+d z_{2} \wedge d \bar{z}_{2}+d z_{3} \wedge d \bar{z}_{3}, \quad F_{2}=d z_{1} \wedge d \bar{z}_{1}+d z_{2} \wedge d \bar{z}_{2}+5 d z_{3} \wedge d \bar{z}_{3},
$$

then the $a_{i}$ 's are traceless with respect to $F_{1}$ and $\left(a_{1}^{2}+a_{2}^{2}\right) \wedge F_{2}=0$. Let $\theta^{j}$ be connection 1-forms such that $d \theta^{j}=\pi^{*} a_{j}$ and define

$$
\omega_{1}=\pi^{*} F_{1}+\theta^{1} \wedge \theta^{2}, \quad \omega_{2}=\pi^{*} F_{2}+\theta^{1} \wedge \theta^{2} .
$$

The 2-forms $\omega_{1}$ and $\omega_{2}$ define respectively a balanced metric $g_{1}$ and an asthenoKähler metric $g_{2}$ on $M$ compatible with the integrable complex structure so that the projection map $\pi$ is holomorphic. $M$ can be alternatively described as the 2-step nilmanifold $G / \Gamma$, where $G$ is the 2-step nilpotent Lie group with structure equations

$$
\left\{\begin{array}{l}
d e^{j}=0, \quad j=1, \ldots, 6 \\
d e^{7}=e^{1} \wedge e^{2}+e^{3} \wedge e^{4}-2 e^{5} \wedge e^{6}, \\
d e^{8}=e^{3} \wedge e^{4}-e^{5} \wedge e^{6}
\end{array}\right.
$$


and $\Gamma$ is a co-compact discrete subgroup, endowed with the invariant complex structure $I$ such that $I e_{1}=e_{2}, I e_{3}=e_{4}, I e_{5}=e_{6}, I e_{7}=e_{8}$. In this setting the 2-form $e^{1} \wedge e^{2}+e^{3} \wedge e^{4}+e^{5} \wedge e^{6}+e^{7} \wedge e^{8}$ defines a balanced metric and $e^{1} \wedge e^{2}+e^{3} \wedge e^{4}+$ $5 e^{5} \wedge e^{6}+e^{7} \wedge e^{8}$ gives an astheno-Kähler metric.

Remark 4.1. The example above has holomorphically trivial canonical bundle and is not simply connected. Other examples on nilmanifolds in every dimension greater or equal to 8 are found by Latorre and Ugarte [42]. More examples could be found on torus bundles with base a Kähler manifold which has known cohomology ring. In the next section we provide such example on a torus bundle over a flag manifold.

\section{BALANCED METRICS ON COMPACT COMPLEX HOMOGENEOUS SPACES WITH INVARIANT VOLUME}

A $2 n$-dimensional manifold $M$ is a complex homogeneous space with invariant volume if there is a complex structure and a nonzero $2 n$-form on $M$ both preserved by a transitive Lie transformation group.

Compact complex homogeneous spaces with invariant volumes have been classified in [37], showing that every compact complex homogeneous space with an invariant volume form is a principal homogeneous complex torus bundle over the product of a projective rational homogeneous space and a complex parallelizable manifold.

A rational homogeneous manifold $Q$ (also called a generalized flag manifold) is by definition a compact complex manifold that can be realized as a closed orbit of a linear algebraic group in some projective space. Equivalently, $Q=S / P$ where $S$ is a complex semisimple Lie group and $P$ is a parabolic subgroup, i.e., a subgroup of $S$ that contains a maximal connected solvable subgroup (i.e. a Borel subgroup). A complex parallelizable manifold is a compact quotient of a complex Lie group by a discrete subgroup.

Chronologically, Matsushima first considered the special case of a semisimple group action, proving in [46] that if $G / H$ is a compact complex homogeneous space with a $G$-invariant volume, then $G / H$ is a holomorphic fiber bundle over a rational homogeneous space and the fiber is a complex reductive parallelizable manifold as a fiber. This kind of fibration is called Tits fibration.

Matsushima's result [46] was improved by Guan in [37]

Theorem 5.1. Every compact complex homogeneous space $M$ with an invariant volume form is a principal homogeneous complex torus bundle

$$
\pi: M \rightarrow G / K \times D
$$

over the product of a projective rational homogeneous space and a complex parallelizable manifold.

In particular, when $M$ is invariant under a complex semisimple Lie group the Tits fibration is a torus fibration. 
Furthermore, in [37] it is shown that the bundle $\pi: M \rightarrow G / K \times D$ arises as a factor of the product of two principal complex torus bundles. One is $\pi_{1}: G / H \rightarrow G / K$, which is the Tits fibration for $G / H$ with fiber tori, and the other is $\pi_{2}: D_{1} \rightarrow D$, where $D_{1}$ is again compact complex parallelizable and the fiber is a complex torus, which is in the center of $D_{1}$. The action for the factor bundle is the anti-diagonal one. The projection $M \rightarrow D$ is the Tits fibration with complex parallelizable fibers.

The structure of $\pi_{1}: G / H \rightarrow G / K$ is discussed in [59]. The compact complex homogeneous spaces $G / H$ with finite fundamental group and $G$ compact are called by Wang [59] $C$-spaces. They fall in two classes Kählerian C-spaces and non-Kählerian C-spaces. The Kählerian C-spaces are well studied and are precisely the generalized flag manifolds or in case $H$ and $K$ are connected, $H=K$. More generally, by [38] a compact complex homogeneous space does not admit a symplectic structure unless it is a product of a flag manifold and a complex parallelizable manifold. In the sequel we'll use the terminology Wang's C-spaces for non-Kählerian C-spaces.

The characteristic classes of $\pi: M \rightarrow G / K \times D$ are $\left(\omega_{1}+\alpha_{1}, \omega_{2}+\alpha_{2}, \ldots, \omega_{2 k}+\alpha_{2 k}\right)$, where $\left(\omega_{1}, \ldots, \omega_{2 k}\right)$ are the characteristic classes of $G / H \rightarrow G / K$ which are $(1,1)$ and $\left(\alpha_{1}, \ldots, \alpha_{2 k}\right)$ are the characteristic classes of $D_{1} \rightarrow D$. Using averaging one can show that there is a unique $G$-invariant representative in each class $\omega_{k}$. The second fibration is a complex torus fibration and its (complex) characteristic classes are of type $(2,0)$ with respect to the complex structure on the base $D$. In particular $\alpha_{i}$ are of type $(2,0)+(0,2)$, i.e. they have representatives of this type.

We start the characterization of the balanced condition with the following observation:

Lemma 5.1. Assume there is an invariant Hermitian metric on the generalized flag manifold with respect to which all traces of the characteristic class $\omega_{i}$ vanish. Then $M$ admits a balanced metric.

Proof. We know that $M$ is the total space of the principal torus fiber construction $\pi: M \rightarrow G / K \times D$. Suppose that $g=g_{1}+g_{2}$ is an invariant Hermitian metric on the base manifold $G / K \times D$ with a fundamental form $F=F_{1}+F_{2}$. In view [1, Theorem 2.2 ] every complex parallelizable manifold has a balanced metric and we may assume $g_{2}$ balanced. Consider the Hermitian metric $g_{M}$ on $M$ defined by

$$
g_{M}:=\pi^{*} g+\sum_{l=1}^{2 k}\left(\theta_{l} \otimes \theta_{l}\right),
$$

where $\theta_{i}$ are connection 1-forms with $J \theta_{2 j-1}=\theta_{2 j}$. Then the fundamental form of the metric $g_{M}$ is given by

$$
F_{M}=\pi^{*} F+\sum_{l=1}^{k}\left(\theta_{2 l-1} \wedge \theta_{2 l}\right) .
$$


From [36, formula (4)] the co-differential of the fundamental form $F_{M}$ on $M$ is given by

$$
\delta F_{M}=\pi^{*}(\delta F)+\sum_{i=1}^{2 k} g\left(F, \omega_{i}+\alpha_{i}\right) \theta_{i}=\pi^{*}(\delta F)+\sum_{i=1}^{2 k} g\left(F, \omega_{i}\right) \theta_{i}
$$

Since $\alpha_{i}$ are of type $(2,0)$ and $(0,2)$, their traces with respect to any $(1,1)$ form vanish. From the formula above, if there is a balanced metric on the generalized flag manifold $G / K$ such that all traces of the classes $\omega_{i}$ vanish, then $M$ admits a balanced metric. The claim follows since every invariant Hermitian metric on a generalized flag manifold is balanced (see Lemma 5.2 below).

By [60, Theorem 8.9] on a reductive homogeneous almost complex manifold $G / K$ such that the isotropy representation of $K$ has no invariant 1-dimensional subspaces, the fundamental form of every invariant almost Hermitian metric is co-closed. This holds, for example, if the isotropy representation is irreducible or if $G$ and $K$ are reductive Lie groups of equal rank. Moreover, Theorem 4.5 in [60] gives a criterion to establish if a reductive homogeneous almost Hermitian manifold $G / K$ is Hermitian. Therefore, applying applying Theorem 8.9 of [60] to the generalized flag manifolds we have

Lemma 5.2. Every invariant Hermitian metric on a generalized flag manifold $G / K$ is balanced.

The main result of this section is:

Theorem 5.2. Let $M$ be a complex compact homogeneous space admitting an invariant volume form and $\pi: M \rightarrow G / K$ be its Tits fibration with $G / K$ rational homogeneous and $\pi_{1}: G / H \rightarrow G / K$ be the associated torus fibration of the Guan's representation of $M$, with characteristic classes $\omega_{1}, \ldots, \omega_{k}$. Then $M$ admits a balanced metric if and only if the span of $\omega_{1}, \ldots, \omega_{k}$ does not intersect the closure of the Kähler cone of $G / K$.

Notice that the first Chern class of a generalized flag manifold always belongs to the Kähler cone and it vanishes if and only if the first Chern class of the base is in the span of $\omega_{i}$. Hence, taking into account [35], we have:

Corollary 5.1. If $M$ admits a balanced metric, then $c_{1}(M) \neq 0$. In particular compact semisimple Lie groups do not admit any balanced metric compatible with Samelson's complex structure.

For the proof of the Theorem we need some facts about the algebraic structure of the generalized flag manifolds and their cones of invariant Hermitian and Kähler metrics.

Let $G$ be a compact semisimple Lie group and $H$ a closed subgroup such that $G / H$ is a complex homogeneous space. Let $\mathfrak{g}$ and $\mathfrak{h}$ be the corresponding Lie algebras 
and $\mathfrak{g}^{c}, \mathfrak{h}^{c}$ their complexifications. As a complex manifold $G / H$ has a useful form of complexification $G^{c} / H^{c}$ where $G^{c}$ and $H^{c}$ are complex Lie groups with Lie algebras $\mathfrak{g}^{c}$ and $\mathfrak{h}^{c}$ and $G$ is a compact real form of $G^{c}$, while $H=H^{c} \cap G$. By a result of Wang [59], there is an inclusion $\mathfrak{h}_{s s}^{c} \subset \mathfrak{h}^{c} \subset \mathfrak{k}^{c}$, where $\mathfrak{k}^{c}$ is a parabolic subalgebra, which is a centralizer of a torus and $\mathfrak{k}_{s s}^{c}=\mathfrak{h}_{s s}^{c}$. Here the subscript " $s s^{\prime}$ denotes the semisimple part. In particular

$$
\mathfrak{h}^{c}=\mathfrak{a}+\mathfrak{h}_{s s}^{c}
$$

where $\mathfrak{a}$ is a commutative subalgebra of some Cartan subalgebra $\mathfrak{t}$ of $\mathfrak{g}^{c}$, which is also the maximal toral subalgebra of $\mathfrak{k}^{c}$. The parabolic algebra $\mathfrak{k}^{c}$ is $\mathfrak{k}^{c}=\mathfrak{t}+\mathfrak{k}_{s s}^{c}$ and is equal to the normalizer of $\mathfrak{h}^{c}$ in $\mathfrak{g}^{c}$. The sum here is not direct because part of $\mathfrak{t}$ is contained in $\mathfrak{k}_{s s}^{c}$. Let $K^{c}$ be a parabolic subgroup if $G^{c}$ with algebra $\mathfrak{k}^{c}$ and $G^{c} / K^{c}$ is the corresponding generalized flag manifold. If $K=G \cap K^{c}$, then the induced map $\pi_{1}: G / H \rightarrow G / K$ is the fibration from above. Fix a system of roots $R \in \mathfrak{t}^{*}$ defined by $\mathfrak{t}$ in $\mathfrak{g}^{c}$. There is also a distinguished set of simple roots $\Pi$ in $R$ which forms a basis for $\mathfrak{t}^{*}$ as a (complex) vector space and defines a splitting $R=R^{+} \cup R^{-}$of $R$ into positive and negative roots.

Now every invariant complex structure on the generalized flag manifold is determined by an ordering of the system of roots of $\mathfrak{g}^{c}$. The complex structure on $G / K$ defines a subset $\Pi_{0}$ in $\Pi$ which corresponds to $\mathfrak{k}^{c}$. This correspondence determines the second cohomology of $G / K$ and we provide some details about it. In general $\mathfrak{k}_{s s}^{c}$ is determined by the span of all roots $R_{0}$ in $R$ which are positive with respect to $\Pi_{0}$. Then the complement $\Pi-\Pi_{0}=\Pi^{\prime}$ provides a basis for the center $\zeta$ of $\mathfrak{k}^{c}$ and there is an identification $\operatorname{span}_{\mathbf{Z}}\left(\Pi^{\prime}\right)=H^{2}(G / K, \mathbb{Z})$. The identification (see for example [2]) is:

$$
\xi \rightarrow \frac{i}{2 \pi} d \xi
$$

where $\xi$ is considered as a left invariant 1-form on $G$ which is a subgroup of $G^{c}$ and $d \xi$ is $a d(\mathfrak{k})$-invariant, hence defines a 2 -form on $G / K$. This form is obviously closed and in fact defines non-zero element in $H^{2}(G / K, \mathbb{Z})$. Moreover every class in $H^{2}(G / K, \mathbb{Z})$ has unique representative of this form. It is known that the Kähler cone can be identified with a positive Weyl chamber determined by the order in $\Pi^{\prime}$.

For the description of the invariant Hermitian metrics on $G / K$ we need first the (reductive) decomposition

$$
\mathfrak{g}^{c}=\mathfrak{k}^{c} \oplus \mathfrak{m}^{c}
$$

where $\mathfrak{m}^{c}=\mathfrak{m}^{+} \oplus \mathfrak{m}^{-}$and $\mathfrak{m}^{ \pm}=\sum_{\alpha \in R^{\prime \pm}} \mathfrak{g}_{\alpha}$. Here $R^{\prime+}$ and $R^{\prime-}$ are the positive and negative roots in $R^{\prime}=R-R_{0}$ and $\mathfrak{g}_{\alpha}$ are the corresponding root spaces. The complex structure on $G / K$ is given by $\left.I\right|_{\mathfrak{m}^{ \pm}}= \pm i I d$. Now let $\mathfrak{m}=\sum \mathfrak{m}_{i}$ be the decomposition of $\mathfrak{m}$ into irreducible components under the action of $\mathfrak{k}^{c}$. Let $B$ be the metric given 
by the negative of the Cartan-Killing form. Any invariant Hermitian metric on $G / K$ is given by positive numbers $\lambda_{i}>0$ as follows:

$$
g=\sum_{i} \lambda_{i} B_{i}
$$

where $B_{i}=\left.B\right|_{\mathfrak{m}_{i}}$. Its fundamental form is $F=\sum_{i} \lambda_{i} F_{i}$ with $F_{i}=B_{i} \circ I$.

It can be written also as

$$
F=\sum_{\alpha \in \mathfrak{m}^{+}}-i \lambda_{\alpha} E_{\alpha}^{*} \wedge E_{-\alpha}^{*}
$$

where $E_{\alpha}$ are unit (and necessary orthogonal) vectors in $\mathfrak{g}_{\alpha}$ and $E_{\alpha}^{*}$ their duals. If $\alpha, \beta \in \mathfrak{m}_{i}$, then $\lambda_{\alpha}=\lambda_{\beta}$. Then the condition $d F=0$ is equivalent (see [3]) to the condition:

For every $\alpha, \beta \in \mathfrak{m}^{+}$with $\alpha+\beta \in \mathfrak{m}^{+}, \lambda_{\alpha}+\lambda_{\beta}=\lambda_{\alpha+\beta}$.

We can summarize the previous observations as:

Lemma 5.3. The cone of the invariant Hermitian metrics (Hermitian cone for short) on the generalized flag manifold $G / K$ is identified with the first octant in the space of all invariant $(1,1)$ forms by assigning to each metric its fundamental form. The cone of the invariant Kähler metrics on the generalized flag manifold $G / K$ is obtained by intersecting the Hermitian cone with the linear subspace of all closed invariant $(1,1)$-forms.

Now we prove Theorem 5.2

Proof of Theorem 5.2. Suppose that the span $C$ of the characteristic classes $\omega_{i}$ does not intersect the Kähler cone. Without loss of generality assume that $\omega_{i}$ are invariant. Then clearly $C$ does not intersect the cone of invariant Hermitian metrics either. From 3 this cone is given by the "first octant" - all $\lambda_{\alpha}$ 's are positive. Clearly a hyperplane does not intersect its closure if and only if its normal vector is in it. Then from Lemma 5.3 there is an element $F^{\prime}=\sum_{\alpha \in \mathfrak{m}^{+}}-i \lambda_{\alpha} E_{\alpha}^{*} \wedge E_{-\alpha}^{*}$ of the Hermitian cone, which is orthogonal with respect to a metric of type (3) above with all $\lambda_{\alpha}=1$, to all $\omega_{i}$. This is equivalent to all $\omega_{i}$ being traceless with respect to $F=\sum_{\alpha \in \mathfrak{m}^{+}}-i \sqrt{\lambda_{\alpha}} E_{\alpha}^{*} \wedge E_{-\alpha}^{*}$. This $F$ is a fundamental form of a balanced metric $g$ on $G / K$ by Lemma 5.2, which by the torus bundle construction gives rise to a balanced metric on $G / H$. Then by Lemma 5.1 we have a balanced metric on $M$.

In the opposite direction, if there is such a class, it defines a non-negative and non-zero form $\alpha$ which pulls back to an exact form on $G / H$. We can also see that its pullback to $M$ is exact too. But as in [47], $0=\int_{M} \pi^{*}(\alpha) \wedge F^{n-1}>0$ for any positive $F$ on $M$ with $d F^{n-1}=0$. So such $F$ does not exist. 
Below we provide an example of a complex compact homogeneous Wang's C-space carrying both balanced and astheno-Kähler metrics. In particular this gives an example of compact simply connected non-Kähler complex manifold admitting balanced and astheno-Kähler metrics, but no SKT metric. More precisely we have:

Proposition 5.1. The homogeneous space $S U(5) / T^{2}$ for appropriate action of $T^{2}$ is simply connected and has an invariant complex structure which admits both balanced and astheno-Kähler metrics, but doesn't admit any SKT metric.

Proof. Consider the flag manifold $S U(5) / T^{4}$. Then a reductive decomposition for this homogeneous space is $\mathfrak{s u}(5)=\mathfrak{t} \oplus \mathfrak{m}$, where $\mathfrak{t}$ is the subspace of traceless diagonal matrices with imaginary entries and $\mathfrak{m}$ is the subspace of skew-Hermitian matrices with vanishing diagonal entries. After complexification, a standard choice of simple roots is the following: let $a_{i j}$ be the basis of $\mathfrak{g l}(5, \mathbb{C})^{*}$ dual to the standard one in $\mathfrak{g l}(5, \mathbb{C})$ containing matrices with 1 at $(i, j)$ th place and all other entries 0 . Then a set of positive roots for $\mathfrak{s l}(5, \mathbb{C})$ is $a_{i j}$ for $i<j$ and $e_{i, i+1}=a_{i i}-a_{i+1 i+1}$, for $1 \leq i \leq 4$. According to the standard theory (see e.g. [13]), the forms $d e_{i, i+1}, 1 \geq i \geq 4$ form a basis of $H^{2}\left(S U(5) / T^{4}\right)$.

Moreover, the complex structure on $\mathfrak{m}^{\mathbb{C}}$ is given by $I\left(a_{i j}\right)=i a_{i j}$ for $i<j$ and $I\left(a_{i j}\right)=-i a_{i j}$ for $i>j$. Denote by $\alpha_{i j}$ the 2 -form $\frac{i}{2} a_{i j} \wedge a_{j i}$. For $i<j$ these are $(1,1)$-forms and the Kähler form for the bi-invariant metric on $S U(5) / T^{4}$ is given by $\sum_{i<j} \alpha_{i j}$

Note that $\left[A_{i j}, A_{j i}\right]=A_{i i}-A_{j j}$, so

$$
d e_{i, i+1}\left(A_{j k}, A_{k j}\right)=-e_{i, i+1}\left(\left[A_{j k}, A_{k j}\right]\right)=-\left(\delta_{i}^{j}-\delta_{i}^{k}\right)+\left(\delta_{i+1}^{j}-\delta_{i+1}^{k}\right) .
$$

Therefore, by computing $d e_{i, i+1}, 1 \geq i \geq 4$, we get that the following four 2 -forms:

$$
\begin{aligned}
& \omega_{1}=2 \alpha_{12}+\alpha_{13}+\alpha_{14}+\alpha_{15}-\alpha_{23}-\alpha_{24}-\alpha_{25}, \\
& \omega_{2}=-\alpha_{12}+2 \alpha_{23}+\alpha_{24}+\alpha_{25}-\alpha_{34}-\alpha_{35}+\alpha_{13}, \\
& \omega_{3}=-\alpha_{13}-\alpha_{23}+2 \alpha_{34}+\alpha_{14}+\alpha_{24}+\alpha_{35}-\alpha_{45}, \\
& \omega_{4}=\alpha_{15}+\alpha_{25}+\alpha_{35}+2 \alpha_{45}-\alpha_{14}-\alpha_{24}-\alpha_{34}
\end{aligned}
$$

form a basis of $H^{2}\left(S U(5) / T^{4}\right)$.

We have that $\omega_{1}+\omega_{2}+\omega_{3}+\omega_{4}$ is weakly positive definite with 3 zero directions and $3 \omega_{1}+5 \omega_{2}+6 \omega_{3}+6 \omega_{4}$ is strictly positive. Now with respect to the bi-invariant metric (which is balanced), the traces of $\omega_{i}$ are all equal to 2. In view of Proposition 3.1 in order to show the existence of an astheno-Kähler metric on $S U(5) / T^{2}$ we need to find two traceless classes. If we consider $F_{1}=\omega_{1}+\omega_{2}-\omega_{3}-\omega_{4}, F_{2}=3 \omega_{1}-\omega_{2}-\omega_{3}-\omega_{4}$ and the strictly positive 2 -form $\Omega=3 \omega_{1}+5 \omega_{2}+6 \omega_{3}+6 \omega_{4}+10\left(\omega_{1}+\omega_{2}+\omega_{3}+\omega_{4}\right)$, we get that $F_{1}^{2} \wedge \Omega^{8}>0$ and $F_{2}^{2} \wedge \Omega^{8}<0$. It is then sufficient to change either $F_{1}$ or $F_{2}$ by a constant to have them satisfying the condition $\left(F_{1}^{2}+F_{2}^{2}\right) \wedge \Omega^{8}=$ 0 . Furthermore $S U(5) / T^{2}$ has a balanced metric (here we can for instance apply Theorem 5.2). Moreover, it does not admit any Kähler structure. 
Note that the forms $\frac{1}{2 \pi i} F_{1}$ and $\frac{1}{2 \pi i} F_{2}$ from above define integer classes and a lot of information about the topology of the space $S U(5) / T^{2}$ as a principal torus bundle over the flag $S U(5) / T^{4}$ can be obtained. In particular it is simply connected and with non-vanishing first Chern class.

Using the obstruction found by Cavalcanti [17, Theorem 5.16] we can now show that $S U(5) / T^{2}$ does not admit any SKT metric, since it can not have symplectic forms. Indeed, it is possible to prove that $h^{3,0}\left(S U(5) / T^{2}\right)$ and $h^{2,1}\left(S U(5) / T^{2}\right)$ both vanish. To calculate the Hodge numbers we use a refinement of the Borel's spectral sequence in 53] which provides an explicit model for the Dolbeault cohomology of principal torus bundles. Recall that a Dolbeault model of a compact complex manifold $M$ is a morphism $\phi:(\Lambda V, \delta) \rightarrow\left(\Omega^{c}(M), \bar{\partial}\right)$ from a commutative differential bi-graded algebra $V=\oplus_{p, q} V^{p, q}, \delta\left(V^{p, q}\right) \subset V^{p, q+1}$, to the Dolbeaut complex of $M$ which preserves the grading and induces an isomorphism on the cohomology. Then Proposition 8 of [53] describes the Dolbeaut model of a torus bundle $M$ over a Kähler base $B$ with $H^{2}(B, \mathbb{C}) \equiv H^{1,1}(B)$ in terms of the de Rham model of $B$. It follows that the Dolbeault cohomology $H^{3,0}\left(S U(5) / T^{2}\right)$ vanishes, because the flag manifold $S U(5) / T^{4}$ has no (2,0)-cohomology. Also any element $\alpha \in H^{2,1}$ has the form $\alpha=\beta \wedge \omega$ with $\beta$ being the $(1,0)$-form and $\bar{\partial} \beta=F_{1}+i F_{2}$ and $\omega=\sum_{i} a_{i} \gamma_{i}$. Then $\bar{\partial} \alpha=$ $\left(F_{1}+i F_{2}\right) \wedge\left(\sum_{i} a_{i} \gamma_{i}\right)$, for some generator $\gamma_{i}$ such that $\sum_{i} \gamma_{i}^{2}+\sum_{i<j} \gamma_{i} \wedge \gamma_{j}=0$. We can check that the quadratic form $\sum_{i} x_{i}^{2}+\sum_{i<j} x_{i} x_{j}$ has maximal rank. However $\left(F_{1}+i F_{2}\right) \wedge\left(\sum_{i} a_{i} \gamma_{i}\right)$ corresponds to a quadratic form of lower rank. So they can not be proportional. This shows that $h^{2,1}=h^{3,0}=0$. On the other side, every class in $H^{2}\left(S U(5) / T^{2}\right)$ is a pullback of a class on the base flag manifold, so it can not have a maximal rank. Hence $S U(5) / T^{2}$ doesn't admit a symplectic structure (this is consistent with [38]).

No general result is known for the existence of SKT metrics on complex homogeneous spaces. Complex parallelizable manifolds cannot admit SKT metrics, by using the argument as in [22] for the invariant case and the symmetrization process in [7, 25, 57]. Indeed, the existence of an SKT metric, implies the existence of a unitary coframe of invariant (1,0)-forms $\left\{\zeta_{i}\right\}$ such that $\bar{\partial} \zeta_{i}=0$, so we can suppose that the fundamental form of the SKT metric is given by $\frac{i}{2} \sum_{i} \zeta_{i} \wedge \bar{\zeta}_{i}$, which cannot be $d d^{c}$-closed. Moreover, by [45] a complex parallelizable manifold cannot admit any astheno-Kähler metric (unless the space is a complex torus). In the next section, we generalize the arguments above so as to characterize SKT Wang C-spaces.

\section{SKT METRICS ON NON-KÄHLER C-SPACES}

Recall that a Wang C-space (or non-Kähler C-space) is a compact complex manifold admitting a transitive action by a compact Lie group of biholomorphisms and finite fundamental group. According to Wang [59], such a space admits a transitive action of a compact semisimple Lie group. The aim of this section is to prove the following 
Theorem 6.1. Every SKT Wang C-space is (up to a finite cover) the product of a compact Lie group and a generalized flag manifold.

Before giving the proof of the theorem we need some preliminary lemmas.

Lemma 6.1. Let $M=G / H$ be a Wang $C$-space. Then $h^{3,0}(M)=0$.

Proof. The Hodge numbers of $M$ can be computed by using the Tanre model [53] for the Dolbeault cohomology of principal torus bundles. As in the proof of Proposition 5.1 we use the Tits fibration $G / H \rightarrow G / K$ and the fact that $h^{2,0}(G / K)$ vanishes. Indeed, by [13, 14.10] $G / K$ is a rational projective algebraic manifold over $\mathbb{C}$ all of whose cohomology is of Hodge type $(p, p)$. Now the Lemma follows from [53], Proposition 8.

Lemma 6.2. Let $M=G / H$ be a Wang $C$-space with $G$ simple. Then $h^{2,1}(M)=0$ unless $H$ is discrete.

Proof. We first consider the case $H$ abelian. In this case $H$ is contained in a maximal torus $T$ and the Tits fibration is $G / H \rightarrow G / T$. From a classical result in [11 the cohomology ring $H^{*}(G / T, \mathbb{C})$ is generated by the products of $\omega_{i}$ 's. The only relations among them are given by $Q_{i}\left(\omega_{1}, \ldots, \omega_{n}\right)=0$, where $Q_{i}\left[x_{1}, \cdots, x_{n}\right]$ are all polynomials invariant under the Weyl group $W_{G}$ of $G$ acting on $\mathbb{R}\left[x_{1}, \cdots, x_{n}\right]$ and the product in $H^{*}(G / T, \mathbb{C})$ is the wedge product of the corresponding representatives. According to a result by Chevalley [18], for a simple Lie group $G$, there exists up to a constant only one $W_{G}$-invariant quadratic polynomial $p \in \mathbb{C}\left[x_{1}, \ldots, x_{n}\right] ; p$ is the polynomial corresponding to the Killing form of the Lie algebra of $G$. In particular, $H^{4}(G / T, \mathbb{C})$ is isomorphic to the space of homogeneous quadratic polynomials factored by $p$. Moreover $p$ is negative definite over real numbers and, consequently, it has a maximal rank over complex numbers.

Assume now that $h^{2,1}(G / H) \neq 0$ and let $\omega_{1}, \omega_{2}, \ldots \omega_{n}$ be the generators of the space $H^{1,1}(G / T, \mathbb{C})$. From Tanre's model it follows that there exists a quadratic relation involving the $\omega_{i}$. Indeed, elements of $H^{2,1}(G / H, \mathbb{C})$ are of the form $\sum_{i} \alpha_{i} \wedge \omega_{i}$, where $\alpha_{i}$ are some vertical $(1,0)$-forms. Let $Q\left(\omega_{1}, \ldots, \omega_{n}\right)=\sum q_{i j} \omega_{i} \omega_{j}$ as above, where $Q$ is a quadratic polynomial in $\mathbb{C}\left[x_{1}, \ldots, x_{n}\right]$.

Since up to a constant there exists only one $W_{G}$-invariant quadratic polynomial $p$ in $\mathbb{C}\left[x_{1}, \ldots, x_{n}\right], Q=c p$ for a constant $c$ and $Q$ has a maximal rank too. But if the Tits fibration has positive-dimensional fiber, then $Q$ depends on $y_{1}, . ., y_{m}$ - variables with $m<n$, where $y_{i}$ 's are linear functions of $x_{i}$ 's. In particular the diagonal form of $Q$ has at most $m$ non-zero entries and it can not have a maximal rank in $\mathbb{C}\left[x_{1}, \ldots, x_{n}\right]$. So the Tits fibration has a discrete fiber and the statement follows.

Now assume $H$ arbitrary. Then the base of the Tits fibration $\pi: G / H \rightarrow G / K$ is a generalized flag manifold and $K$ contains a maximal torus $T$. A result by BernsteinGelfand-Gelfand in [8] implies that $\pi^{*}: H^{*}(G / K, \mathbb{C}) \rightarrow H^{*}(G / T, \mathbb{C})$ is injective. Moreover, the image of $\pi^{*}$ consists in the $W_{K^{-}}$invariant elements of $H^{*}(G / T, \mathbb{C})$. So, 
if $\omega_{1}, \ldots, \omega_{k}$ are the characteristic classes of the Tits fibration, then $\pi^{*}\left(\omega_{i}\right)$ belongs to $H^{1,1}(G / T, \mathbb{C})$ and if $Q=\sum q_{i j} \omega_{i} \omega_{j}=0$, then $\pi^{*}(Q)=0$ in $H^{1,1}(G / T, \mathbb{C})$. But then there will be additional quadratic relation among the set of the $W_{K}$-invariant polynomials in $\mathbb{C}\left[x_{1}, \ldots x_{n}\right]$. When $W_{K}$ is non-trivial this is impossible. So, $H$ has to be either abelian or discrete and, from the first part of the proof, it has to be discrete.

Lemma 6.3. Let $M=G / H$ be a Wang $C$-space as above, but with $G$ semisimple. Then $h^{2,1}(M) \neq 0$ only if $M$ has a finite cover which is biholomorphic to a product of a compact even-dimensional Lie group and another Wang C-space.

Proof. As in the previous proof, the assumption leads to the existence of a quadratic relation $Q$ on the characteristic classes of the Tits fibration $\pi: G / H \rightarrow G / K$. This time the base $G / K$ is a generalized flag manifold with $G$ a semisimple Lie group, so by [12, Corollary, p. 1148] it is a product of generalized flag manifolds $G_{1} / K_{1} \times . . \times G_{k} / K_{k}$ with $G_{i}$ simple. Now we identify the elements of $H^{4}(G / K, \mathbb{C})$ with the $W_{K}$-invariant quadratic polynomials in $\mathbb{C}\left[x_{1}^{(1)}, . ., x_{s_{1}}^{(1)}, \ldots, x_{1}^{(k)}, . ., x_{s_{k}}^{(k)}\right]$, where $x_{1}^{(i)}, . ., x_{s_{i}}^{(i)}$ correspond to the generators of $H^{1,1}\left(G_{i} / T_{i}, \mathbb{C}\right)$ and $T_{i}$ is the maximal tori in $K_{i}$. This follows because the maximal torus of $K$ is the maximal torus of $G$ and it is the product $T=T_{1} \times \cdots \times T_{k}$ of maximal tori $T_{i}$ of $K_{i}$, which are also maximal in $G_{i}$. Then the polynomial corresponding to $Q$ is of the type $Q=c_{1} p_{1}+\ldots+c_{k} p_{k}$ for some constants $c_{i}$, where $p_{i}$ is the unique (up to a constant) quadratic $W_{K_{i}}$-invariant polynomial depending on the variables corresponding to the classes of $H^{1,1}\left(G_{i} / T_{i}, \mathbb{C}\right)$. Suppose now that $c_{1} \neq 0, c_{2}=\ldots=c_{k}=0$. Then $Q$ is a function of only the variables $x_{1}^{(1)}, \ldots, x_{s_{1}}^{(1)}$ and has a maximal rank. As a consequence the Tits fibration has as fiber the torus $T_{1}$ and characteristic classes only in $H^{1,1}\left(G_{1} / T_{1}, \mathbb{C}\right)$ so $K_{1}$ is abelian and since $T_{1}$ is maximal abelian, $K_{1}=T_{1}$. Moreover as in the previous Lemma, we have $(1,0)$ forms $\alpha_{1}, \alpha_{2}, \ldots, \alpha_{s_{1}}$ such that $\bar{\partial}\left(\sum \alpha_{i} \wedge \omega_{i}^{1}\right)=Q\left(\omega_{1}^{1}, \ldots, \omega_{s_{1}}^{1}\right)$. In particular the real and imaginary parts of $\alpha_{i}^{1}$ are all in $\mathfrak{t}_{1}^{*}$, where $\mathfrak{t}_{1}$ is the Lie algebra of $T_{1}$. The the restriction of the Tits bundle over $G_{1} / K_{1}=G_{1} / T_{1}$ is (up to a finite cover) $G_{1} \rightarrow G_{1} / T_{1}$ and the tangent bundle of $G_{1}$ is complex-invariant, so $M$ is biholomorphic (up to a finite cover) to the product $G_{1} \times\left(G_{2} \times \ldots \times G_{k}\right) / \bar{H}$, where $\bar{H}$ contains $K_{2} \times \ldots \times K_{k}$.

Suppose now that more than one of $c_{i}$ 's is non-zero. Then $Q$ depends only on the variables, corresponding to the nonvanishing $c_{i}$ and has maximal rank in them. So again, using the same considerations as above, $M$ is up to a finite cover the product of $G_{i}$ 's for these $i$ and some Wang C-space invariant under the action of the product of $G_{j}$ 's corresponding to the $c_{j}$ 's with $c_{j}=0$.

Proof of Theorem 6.1. Every Wang C-space $M$ is represented as $M=G / H$ for a compact semisimple Lie group $G$. Now assume that $M$ admits an SKT metric but it has no Kähler metrics. From a result by Borel [12] (see also [61, Theorem 5.8 ) $M$ does not admit an invariant symplectic form. By averaging, $M$ does not admit any symplectc structure. Then by Cavalcanti's result [17] about the non-existence 
of SKT and non-Kähler metrics on a compact complex manifold and Lemma 6.1 we have $h^{2,1}(M) \neq 0$. By Lemma 6.3, $M$ is biholomorphic (up to a finite cover) to a product $G_{1} \times M_{1}$, where $M_{1}$ is another Wang C-space. We can embed $M_{1}$ into (possible finite cover of ) $M$ as a complex submanifold and note that a restriction of an SKT metric to a complex submanifold is again SKT, we obtain an SKT metric on $M_{1}$. If $M_{1}$ admits no Kähler metric again, we can continue until we get a factor which a C-space, but it admits a Kähler metric, or is empty.

Remark 6.1. A product of a Kähler space and an SKT space is SKT. Since the generalized flag manifolds admit Kähler structures (and they are the only homogeneous manifolds of compact semi-simple Lie groups which do, by [12]), the spaces of Theorem 6.1 admit SKT metrics.

Note also that on the product of $G_{i}$ for the $i$ with $c_{i} \neq 0$ in the proof, the complex structure does not have to be a product of complex structures on $G_{i}$. This is the case of $S^{3} \times S^{3}$ for example.

Acknowledgments. The work on this project started with the second named author's visit to the University of Torino. He is grateful to Mathematics Department there, as well as Max Plank Institute (Bonn) and Institute of Mathematics and Informatics of the Bulgarian Academy of Sciences for the hospitality and support during the preparation of parts of this paper. We would like to thank Valentino Tosatti for suggesting the problem of the existence of an astheno-Kahler metric on a compact balanced non-Kähler complex manifold. Moreover, we are grateful to Liana David and Luis Ugarte for useful comments on the paper. We would like also to thank the anonymous referees for useful comments.

\section{REFERENCES}

[1] E. Abbena, A. Grassi, Hermitian left invariant metrics on complex Lie groups and cosymplectic Hermitian manifolds, Boll. Un. Mat. Ital. A (6) 5 (1986), no. 3, 371-379.

[2] D. Alekseevsky, Flag manifolds, Zb. Rad. (Beogr.) 6 (14) (1997), 3-35.

[3] D. Alekseevsky, L. David, A note about invariant SKT structures and generalized Kähler structures on flag manifolds, Proc. Edinb. Math. Soc. (2) 55 (2012), 543-549.

[4] B. Alexandrov, G. Grantcharov, S. Ivanov, Curvature properties of twistor spaces of quaternionic Kähler manifolds, J. Geometry 62 (1998), 1-12.

[5] B. Alexandrov, S. Ivanov, Vanishing theorems on Hermitian manifolds, Differential Geom. Appl. 14 (2001), no. 3, 251-265.

[6] L. Alessandrini, Classes of compact non-Kähler manifolds, C. R. Math. Acad. Sci. Paris 349 (2011), 1089-1092.

[7] F. Belgun, On the metric structure of non-Kähler complex surfaces, Math. Ann. 317 (2000), $1-40$.

[8] I. N. Bernstein, I. M. Gelfand, and S. I. Gelfand, Schubert cells and cohomology of the space G/P, Russian Math. Surveys 28 (1973), 1-26.

[9] A. L. Besse, Einstein manifolds, Springer-Verlag, Berlin, 1987. 
[10] J. M. Bismut, A local index theorem for non-Kähler manifolds, Math. Ann. 284 (1989), 681-699.

[11] A. Borel, Sur la cohomologie des espaces fibrs principaux et des espaces homognes de groupes de Lie compacts, Ann. of Math. 57 (1953), 115-207.

[12] A. Borel, Kählerian coset spaces of semi-simple Lie groups, Proc. Nat. Acad. Sci. 40 (1954), $1147-1151$.

[13] A. Borel, F. Hirzebruch, Characteristic classes and homogeneous spaces. I and II Amer. J. Math. 80 (1958), 458-538 and 81 (1959), 315-382.

[14] A. Borel, R. Remmert, Über kompakte homogene Kählersche Mannigfaltigkeiten. Math. Ann. 145 1961/1962, 429-439.

[15] F. Campana, On twistor spaces of the class C, J. Differential Geom. 33 (1991), 541-549.

[16] J. A. Carlson, D. Toledo, On fundamental groups of class VII surfaces, Bull. London Math. Soc. 29 (1997), 98-102.

[17] G. R. Cavalcanti, Hodge theory and deformations of SKT manifolds, arXiv:1203.0493,

[18] C. Chevalley, Invariants of finite groups generated by reflections, Amer. J. Math. 77 (1955), 778-782.

[19] I. Chiose, Obstructions to the existence of Kähler structures on compact complex manifolds. Proc. Amer. Math. Soc. 142 (2014), no. 10, 3561-3568.

[20] I. Chiose, R. Rasdeaconu, I. Suvaina, Balanced Manifolds and SKT Metrics, arXiv:1608.08721.

[21] G. Deschamps, N. Le Du, C. Moirougane, Hessian of the natural Hermitian form on twistor spaces, Bull. Soc. Math. France 145 (2017), no. 1, 1-7.

[22] A. Di Scala, J. Lauret, L. Vezzoni, Quasi-Kähler Chern-flat manifolds and complex 2-step nilpotent Lie algebras, Ann. Sc. Norm. Super. Pisa Cl. Sci. (5) 11 (2012), 41-60.

[23] N. Enrietti, A. Fino, L. Vezzoni, Tamed Symplectic forms and Strong Kähler with torsion metrics, J. Symplectic Geom. 10 (2012), 203-223.

[24] T. Fei, Construction of Non-Kähler Calabi-Yau Manifolds and new solutions to the Strominger System, Adv. Math. 302 (2016) 529550.

[25] A. Fino, G. Grantcharov, On some properties of the manifolds with skew symmetric torsion and special holonomy, Adv. Math. 189 (2004), 439-450.

[26] A. Fino, M. Parton, S. Salamon, Families of strong KT structures in six dimensions, Comment. Math. Helv. 79 (2004) 317-340.

[27] A. Fino, A. Tomassini, Blow-ups and resolutions of strong Kähler with torsion metrics, Adv. Math. 22 (2009), 914-935.

[28] A. Fino, A. Tomassini, On astheno-Kähler metrics, J. London Math. Soc. 83 (2011), 290-308.

[29] A. Fino, L. Vezzoni, Special Hermitian metrics on compact solvmanifolds, J. Geom. Phys. 91 (2015), 40-53.

[30] A. Fino, L. Vezzoni, On the existence of balanced and SKT metrics on nilmanifolds, Proc. Amer. Math. Soc. 144 (2016), 2455-2459.

[31] J. Fu, J. Li and S.-T. Yau, Balanced metrics on non-Kähler Calabi-Yau threefolds. J. Differential Geom. 90 (2012), 81-129.

[32] J. Fu, Z. Wang, D. Wu, Form-type Calabi-Yau equations, Math. Res. Lett. 17 (2010), no. 5, 887-903.

[33] S. J. Gates, Jr., C. M. Hull, M. Roček, Twisted multiplets and new supersymmetric nonlinear $\sigma$-models, Nuclear Phys. B 248 (1984), 157-186.

[34] P. Gauduchon, La 1-forme de torsion dune variété hermitienne compacte, Math. Ann. 267 (1984), 495-518.

[35] G. Grantcharov, Geometry of compact complex homogeneous spaces with vanishing first Chern class, Adv. Math. 226 (2011), 3136-3159. 
[36] D. Grantcharov, G. Grantcharov, Y. S. Poon, Calabi-Yau connections with torsion on toric bundles, J. Differential Geom. 78 (2008), no. 1, 13-32.

[37] D. Guan, Classification of compact complex homogeneous spaces with invariant volumes, Trans. AMS 354 (2002), 4493-4504.

[38] D. Guan, A splitting theorem for compact complex homogeneous spaces with a symplectic structure, Geom. Dedicata 63 (1996), 217-225.

[39] R. Harvey, B. Lawson, An intrinsic characterization of Kähler manifolds, Invent. Math. 74 (1983), no. 2, 169-198.

[40] J. Jost, S-T. Yau, A non-linear elliptic system for maps from Hermitian to Riemannian manifolds and rigidity theorems in Hermitian geometry, Acta Math. 170 (1993), 221-254; Corrigendum Acta Math. 173 (1994), 307.

[41] D. Kaledin, M.Verbitsky, Non-Hermitian Yang-Mills connections, Selecta Math. New Series 4 (1998), 279-320.

[42] A. Latorre, L. Ugarte, On non-Kähler compact complex manifolds with balanced and asthenoKähler metrics, C. R. Math. Acad. Sci. Paris 355 (2017), no. 1, 9093. .

[43] J. Li, S.-T. Yau, F. Zheng, On projectively flat Hermitian manifolds, Commun. Anal. Geom. 2 (1994), 103-109.

[44] K. Liu, X. Yang, Hermitian harmonic maps and non-degenerate curvatures, Math. Res. Lett. 21 (2014), no. 4, 831-862.

[45] K. Matsuo, Astheno-Kähler structures on Calabi-Eckmann manifolds, Colloq. Math. 115 (2009), no. $1,33-39$.

[46] Y. Matsushima, Sur Certaines Varietes Homogenes Complexes, Nagoya Math. J. 18 (1961), $1-12$.

[47] M.L. Michelsohn, On the existence of special metrics in complex geometry, Acta Math. 143 (1983), 261-295.

[48] M. Pontecorvo, Complex structures on quaternionic manifold, Diff. Geom Appl. 4 (1994), 163177.

[49] H. Samelson, A class of complex-analytic manifolds, Portugaliae Math. 12 (1953), 129-132.

[50] A. Strominger, Superstrings with torsion, Nuclear Phys. B 274 (1986), 253-284.

[51] A. Swann, Twisting Hermitian and hypercomplex geometries, Duke Math. J. 155, (2010), 403431.

[52] G. Székelyhidi, V. Tosatti, B. Weinkove, Gauduchon metrics with prescribed volume form, arXiv: 1503.04491

[53] D. Tanre, Modele de Dolbeaut et fibre holomorphe, J. Pure Appl. Algebra 31 (1994), 333-345.

[54] C. H. Taubes, The existence of anti-self-dual conformal structures, J. Differential Geom. 36 (1992), no. 1, 163-253.

[55] A. Tomberg, Twistor spaces of hypercomplex manifolds are balanced, Adv. Math. 280 (2015), $282-300$.

[56] V. Tosatti, B. Weinkove, Hermitian metrics, $(n-1, n-1)$ forms and Monge-Ampére equations, arXiv:1310.6326, to appear in J. Reine Angew. Math.

[57] L. Ugarte, Hermitian structures on six dimensional nilmanifolds, Transf. Groups 12 (2007), $175-202$.

[58] M. Verbitsky, Rational curves and special metrics on twistor spaces, Geometry and Topology 18 (2014), 897-909.

[59] H.C. Wang, Closed manifolds with homogeneous complex structure, Am. J. Math. 76 (1954), $1-32$.

[60] J. Wolf, A. Gray, Homogeneous spaces defined by Lie groups automorphisms. II, J. Differential Geom. 2 (1968), 115-159. 
[61] P. B. Zwart, William M. Boothby, On compact homogeneous symplectic manifolds, Annales de l'institut Fourier 30 (1980), 129-157.

Dipartimento di Matematica "Giuseppe Peano", Università di Torino, Via Carlo Alberto 10, 10123 Torino, Italy

E-mail address: annamaria.fino@unito.it, luigi.vezzoni@unito.it

Department of Mathematics and Statistics Florida International University, MiAMI FLORIDA, 33199, USA

E-mail address: grantchg@fiu.edu 